\title{
Recovery Phase of the Murine Rod Photoresponse Reconstructed from Electroretinographic Recordings
}

\author{
Arkady L. Lyubarsky and Edward N. Pugh, Jr. \\ Department of Psychology and institute of Neurological Sciences, University of Pennsylvania, \\ Philadelphia, Pennsylvania 19104-6196
}

\begin{abstract}
The activation and recovery phases of the murine rod photoresponse were determined from corneal electroretinograms (ERGs) obtained in response to pairs of full-field flashes producing $50-10^{5}$ photoisomerized rhodopsins $\left(\mathrm{R}^{*}\right)$ per rod. The a-wave component of the ERG in response to the initial flash provided a well established measure of the activation phase of the rod response. The amplitude of the a-wave response to an intense second flash $\left(45,000 \mathrm{R}^{*}\right)$ delivered $0.2-5$ seconds (s) after the first flash was used to reconstruct the recovery phase of the response. For $160-3000 R^{*}$ rod $^{-1}$, recovery curves were isomorphic, translating on the time axis such that each e-fold increase in $\mathrm{R}^{*}$ produced an incremental recovery delay of $\tau_{\mathrm{c}}=$ $210 \pm 50 \mathrm{~ms}$ (mean $\pm \mathrm{SD}$ ). For initial flashes producing $>3000$ $R^{*}$, recovery curves lost their initial isomorphism and half-times
\end{abstract}

had intensity dependence exceeding $1 \mathrm{~s}$ per e-fold increase in $R^{\star}$. We conclude that for flashes producing $<3000 R^{*}$, the effective lifetime of these $\mathrm{R}^{*}$ is not $>210 \mathrm{~ms}$. Two extant and non-mutually exclusive hypotheses are discussed that can account for the sharp increase in recovery times from flashes producing $>3000 R^{*}$. They are as follows: (1) $\sim 0.03 \%$ of $R^{*}$ have a lifetime exceeding $1 \mathrm{~s}$; and (2) the $\gamma$ subunit of phosphodiesterase $\left(\mathrm{PDE}_{\gamma}\right)$ serves as a GTPase-activating factor, and 3000 $\mathrm{R}^{*}$ produce sufficient activated $\mathrm{G}$-protein $\left(\mathrm{G}^{*}\right)$ to exceed the total quantity of $\mathrm{PDE}_{\gamma}$ subunits such that excess $\mathrm{G}^{*}$ must wait for unoccupied $P D E_{\gamma}$ to inactivate via GTP hydrolysis.

Key words: phototransduction; rod photoreceptors; photoresponse recovery; electroretinogram; murine rod responses; inactivation
The biochemical events underlying the activation of the phototransduction cascade in vertebrate photoreceptors are well established (Pugh and Lamb, 1993; Yau, 1994; Hofmann, in press). Moreover, recent investigations have shown that models of activation based on the established biochemistry can provide quantitatively accurate accounts of the amplification and kinetics of the electrical responses of individual vertebrate rods (Lamb and Pugh, 1992; Kraft et al., 1993; Pugh and Lamb, 1993) and of the a-wave of the human electroretinogram (ERG) (Breton et al., 1994; Hood and Birch, 1994), which is proportional to the activation phase of the rod photocurrent (Hagins et al., 1970). Thus, much of the current research in the area of phototransduction is focused now on characterizing the recovery phasc of photoresponses and on linking measured photoresponse recoveries to underlying biochemical events.

A particularly salient feature of the recovery phase of the flash responses of many rods is that they are approximately isomorphic over a substantial intensity range, from flash intensities that just saturate the response to magnitudes one or more log units higher. Moreover, the isomorphic response recoveries translate on the time axis with a characteristic linear time increment per geometric increase in photoisomerizations (Hodgkin et al., 1984; Pepperberg et al., 1992). This translatory behavior of the recovery phase of individual rods is consistent with the hypothesis that the isomor-

\footnotetext{
Recejved Aug. 14, 1995; revised Sept. 28, 1995, accepted Oct. 2, 1995.

This work was supported by National Institutes of Health Grant EY-02660 and by the University of Pennsylvania Treatment Initiative for Herediary Retinal Degeneration. We thank Drs. Jean Bennet and Alan Latics of the Ophthalmology Department of the University of Pennsylvaniza for advice and support, Stacey Weinsteith for able assistance, and Dr. J. Schnapf for providing data used in Figure 4.

Correspondence should be addressed to E. N. Pugh, Jr., Depariment of Psychology, University of Pensylvania, 3815 Walnut Street, Phiładelphia, PA 19104-6196. Copyright 1996 Society for Neuroscience 0270-6474/96/160563-09\$05.00//0
}

phic recoveries reveal that the transduction mechanism--both activation and inactivation-preceding cGMP hydrolysis is responding linearly to light, and that an overall "effective" or "dominant" time constant for the linear process can be estimated from the increase in recovery time per e-fold increase in flash intensity (Baylor et al., 1974; Adelson, 1982; Pepperberg et al., 1992). Based on bovine light-scattering signals attributable to G-protein activation, on recordings from amphibian rods and, recently, on the analysis of human ERGs, it has been argued that the dominant time constant corresponds to the inactivation time of the cnzymatically active form of rhodopsin, having an effective lifetime of $\sim 2$ seconds (s) (Pepperberg et al., 1992; Birch cl al., 1995).

Rodent retinas have been a mainstay of mammalian retinal research for some time and, indecd, it was the work of Hagins et al. (1970) and Penn and Hagins (1972) on albino-rat retinal slices that established the existence of the dark circulating currents of rods. That same work also enunciated the hypothesis that the blockage of the rod circulating current generated the field potential known as the a-wave of the ERG. With the availability of mice having knock-outs of, or transgenes for, proteins implicated in the phototransduction cascade inactivation (Chen et al., 1995; Goto et al., 1995), the utility of having a method of measuring rod response recovery kinetics noninvasively in mice is greatly increased. Thus, in this investigation we have pursued the goal of characterizing both the activation and inactivation phases of murine rod photoresponses by measuring the a-wave of the ERG in response to individual and paired flashes.

\section{MATERIALS AND METHODS}

Origin of the murine ERG a-wave in the circulating current of the rods

An abundance of work, including the seminal work of Hagins ct al. (1970) and Penn and Hagins (1972), hats demonstrated that the a-wave of the 
ERG of rodents is dominated by the responses of rods. Recent evidence strongly supports the view that the a-wave is directly proportional to the rod photocurrent, which has allowed the kinetics of the activation phase of the rod response to be characterized in vivo. Accepting that the a-wave is a linear monitor of the magnitude of the photocurrent, one also can reason that the a-wave amplitude could provide a measure of the recovery phase of the rod response even in the presence of other ERG components. The appropriate measure is the amplitude of response to a second, a-wave-saturating flash, which should monitor the degree of recovery of the circulating current from the first flash. Birch et al. (1995) recently have used this measure in an analysis of human ERGs.

One necessary condition for our double-flash protocol to be a valid monitor of the rod photoresponse is that there be no significant cone contribution to the murine a-wave. The credibility of this assumption is based on the following experimental facts. First, the number of cones in the mouse retina is known not to exceed 3\% (Carter-Dawson and LaVail, 1979). Because the a-wave amplitude is proportional to the amplitude of photocurrent, it is unlikely that its cone component exceeds several percent.

Second, transgenic mice with three point mutations in opsin (V20G, $P 23 H, P 27 L$ ) exhibit a slow degeneration of rods (half-life, $\sim 1$ month) followed by much slower degeneration of cones (half-life, $\sim 6$ months). ERG recordings from these animals show that during their life the amplitudes of both the a-wave and the rod b-wave (fully dark-adapted, very dim flashes) decline with the time course of retinal rhodopsin content, whereas the cone function monitored by the amplitude of the cone b-wave (light-adapted) remains relatively unscathed (>50\% compared with control) even after the loss of $>90 \%$ of the a wave and rhodopsin (Goto et al., 1995).

Third, cones are known to have much faster kinetics of recovery than rods. ERG recordings of live cynomologus monkeys, the retinas of which contain strong proportions of both cones and rods, show that for light stimuli causing photoisomerization of several thousand visual pigment molecules per cell, recovery of cone photopotentials occurs within $50 \mathrm{~ms}$ (Brown et al., 1965). Single-cell photocurrent data from macaque cones show recovery to flashes of such intensity to be complete in $100-150 \mathrm{~ms}$ (Schnapf et al., 1990). Because the minimal interstimulus interval in our double-flash experiments was $200 \mathrm{~ms}$, any cone contribution to the a-wave should have presented at this time. However, the ERGs in our doubleflash experiments did not exhibit any detectable a-wave component during the time periods after the first flash when rods were expected to be saturated, based on published photocurrent data (compare Figs. 5, 6). Thus, we conclude that our murine a-wave data reflect the responses of rods alone.

Another condition necessary for a double-flash protocol to yield accurate estimates of the fraction of residual rod circulating current at various times after an initial flash is that the second flash be sufficiently intense to saturate the a-wave. Because the b-wave intrudes and truncates the a-wave (compare Fig. 2), meeting this second condition requires that the second probe flash be much more intense than required merely to saturate the photocurrent itself. This condition is examined further after presentation of relevant light-intensity calibration information (see $\mathrm{Ex}$ pcrimental protocols).

\section{ERG recording and optical stimulation}

All experimental procedures were done in compliance with National Institutes of Health guidelines, as approved by the University of Pennsylvania's Institutional Animal Care and Use Committee. Experimental animals normally were pigmented 10 - to 16 -week-old $\mathrm{CBA} / \mathrm{CAJ}$ mice weighing $25-35 \mathrm{gm}$. The animals were dark-adapted overnight and anesthetized before the experiment with an intraperitoneal injection of a saline solution containing (in $\mu \mathrm{g} / \mathrm{gm}$ body weight): 15 ketamine, 6 xylazine, and 600 urethane. These dosages were adopted from Goto et al. (1995). The pupil was dilated with $1 \%$ tropicamide saline solution (Mydriacil, Alconox, New York, NY), and the anesthetized animals were held on a heating plate maintaincd at $38^{\circ} \mathrm{C}$ throughout the experiment in a light-tight Faraday cage.

ERGs were recorded via a coiled, $0.2 \mathrm{~mm}$ platinum wire making electrical contact with the cornea through a layer of methylcellulose solution (Goniosol, Iolab Pharmaceuticals, Indianapolis, IN). A tungsten electrode placed in the mouth served as both reference and ground. Responses were amplified differentially 1000 - to 5000 -fold, bandpassfiltered at $0.1-1000 \mathrm{~Hz}$ (2-pole Butterworth filters), digitized with a 12 bit analog-to-digital converter at 2 or $5 \mathrm{kHz}$, and displayed and stored with a Digidata $\mathrm{PC}$ interface and Axotape2 software (Axon Instruments, Foster City, CA).

A two-channel optical apparatus delivered the light stimulation. Each channel consisted of a xenon flash unit, a circular neutral-density wedge, filter holders, and focusing lenses. One xenon flash unit had a flash duration of - $20 \mu \mathrm{s}$ (3P4 short are lamp, EG\&G Elcctrooptics, Salcm, MA), and the other had a flash duration of $\sim 1 \mathrm{~ms}$ (Vivitar Photoflash, Vivitar, Santa Monica, CA). The light beams from the two channels were combined by a beam splitter and focused on the end of a fiberoptic light guide that delivered the light into the Faraday cage.

Full-field stimulation of the mouse's retina was achieved via a hemispherical diffusing element fitted to the termination of the light guide; this diffusing element created a ganzfeld and is illustrated in Figure 1 (Frishman and Steinberg, 1989). The hemispherical diffusing element had the recording electrode embedded in its outer rim and was pressed gently against the eye, which was covered first with a thick layer of methylcellulose solution that provided protection for the eye and electrical contact.

The optical channel with the $20 \mu$ s flash unit was used for single-flash experiments and for the initial test flash in the two-flash stimulation paradigm. The channel with the $1 \mathrm{~ms}$ flash was used to deliver the second flash that probed the residual a-wave amplitude in the two-flash experiments. The intensity of the light stimuli was controlled by stepping motors, which positioned the neutral density wedges (Inconel, Kodak, Rochester, NY), and/or by Inconel glass neutral-density filters. The filters and wedges were calibrated with monochromatic light. Stimuli were rendered monochromatic via interference filters with $8-10 \mathrm{~nm}$ bandpass and were blocked outside the bandpass (Baird-Atomic, Boston, MA). The timing of flash stimulation and the wedge positions were controlled by a dedicated PC/AT-class computer with customized software written in Microsoft Quick Basic.

\section{Light calibrations and estimation of photoisomerizations per rod per flash}

The absolute irradiance (in photons $/ \mathrm{mm}^{2}$ ) produced by the diffusing element at the plane of the mouse's pupil was measured with a Pin-10 photodiode (United Detector Technology, Waltham, MA); the photodiode was operated in photovoltaic mode and had been calibrated absolutely by the National Research Council of Canada. For the diffusing element to create a uniform illumination of the retina, the quantal flux entering the pupil from different angles must be uniform. To determine the angular dispersion of the light emerging from the diffusing element, a special tubular probe was made and fitted to the photodiode; this probe limited the angular subtense of the light it collected to $<2^{\circ}$. Measurements made with this probe are illustrated in Figure 1, $C$ and $D$, and show that for the region of the diffusing element that surrounded the mouse's pupil, the intensity was approximately isotropic at that plane. Calibrations of the irradiance at the pupillary plane were made regularly throughout the period of experimentation.

All flash intensities in this paper are reported in units of photoisomerizations per rod $(\Phi)$. Our estimation of $\Phi$ assumed that all photons entering the mouse's pupil either are lost in transmission or are distributed uniformly over the photopigment layer of the retina. We computed $\Phi$ with the following formula:

$$
\Phi=Q(\lambda) \tau(\lambda) \frac{S_{\text {pupil }}}{S_{\text {rclina }}} a_{C}(\lambda),
$$

where $\lambda$ is the wavelength of monochromatic light, $Q(\lambda)$ is the measured photon density at the pupil plane, $\tau(\lambda)$ is the transmission coefficient of the ocular media and neural retina, $S_{\text {pupil }}$ is the area of the dilated pupil, $S_{\text {retint }}$ is the surface area of the retina, and $a_{\mathrm{C}}(\lambda)$ is the collecting area of an individual rod.

Values for the parameters in Equation 1 were taken from the literature on mouse and rat as follows. Alpern et al. (1987) estimated that $\tau(500$ $\mathrm{nm})=0.68$ for the albino rat. If, as reported by Alpern et al. (1987) for the rat, most transmission loss occurs in the neural retina of the mouse, then despite the much smaller lens we would expect $\tau(500 \mathrm{~nm})$ to be only slightly higher in the mouse; thus, we assumed $\tau(500 \mathrm{~nm})=0.7$. Based on the data of Remtulla and Hallett (1985) and our own observations, the fully dilated pupil of the adult mouse was taken to have a diameter of 2 $\mathrm{mm}$; thus, we obtained $S_{\text {pupil }}=3.14 \mathrm{~mm}^{2}$. Again, based on the data of eye size by Remtulla and Hallett (1985), we took the neural retina at the level at which light guiding into the receptors occurs to be a hemisphere of radius of $1.5 \mathrm{~mm}$; the total surface area of the retina, $S_{\text {retina }}$ thus was estimated to be $14.1 \mathrm{~mm}^{2}$. 
A

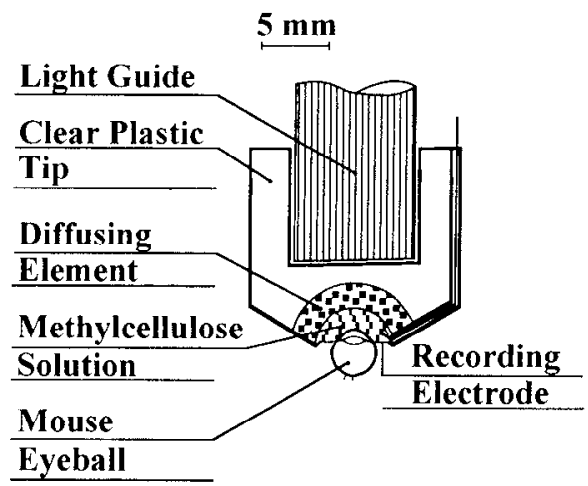

B

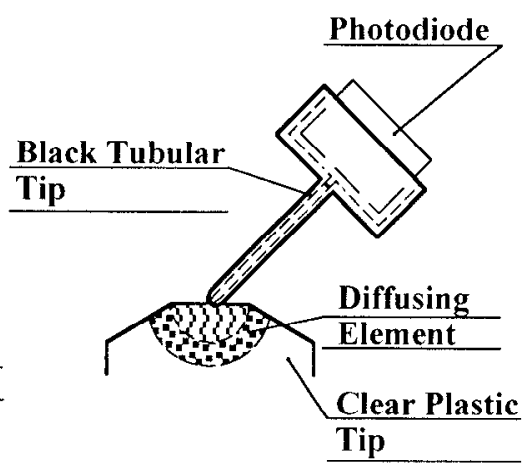

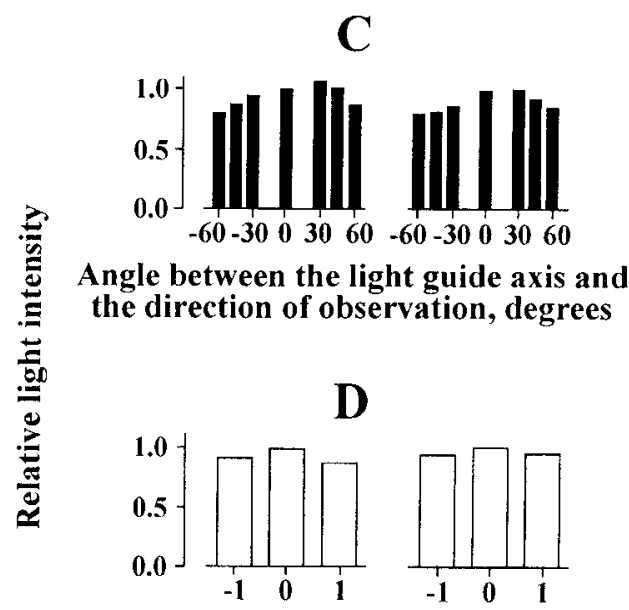

Distance from the light guide axis, $\mathrm{mm}$

Figure 1. Design of the optical system and tests of illumination isotropy. A, Design of the full-field illuminating system. Light emitted through the end of a 7-mm-diameter fiber optic light guide was diffused isotropically by a hemispherical element glued onto the end of a clear plastic tip. The diffusing element ( $6 \mathrm{~mm}$ inner diameter) with the recording electrode $(0.2 \mathrm{~mm}$ coiled platinum wire) was pressed gently against the mouse eye. The space between the cornea and the hemispherical element was filled with high-viscosity methylecllulose solution providing both protection and electrical contact with cornca, $B$, Diagram illustrating light calibration control experiments. The intensity of light emitted at different dircetions was measured by a photodiode with a tubular probe liniting the diode angular subtense to $\sim 2^{\circ}$. The tip of the probe was placed in the area of the mouse pupil. $C$ and $D$, Calibration measurements testing illumination isotropy. C, Light intensity measured in the center of the diffusing hemisphere at different angles in two perpendicular planes. The $0^{\circ}$ angle corresponds to the probe situated along the light guide axis. $D$, Light intensity measured with the probe situated at $0^{\circ}$ and translated with respect to the center of the diffusing element in two perpendicular planes.

We estimated $a_{C}(\lambda)$ in two slightly different ways. The first way used the following formula:

$$
a_{(}(\lambda)={\frac{\left[1-10^{D(\lambda)}\right] \gamma}{\sigma}}^{\sigma}
$$

where $D(\lambda)$ is the in situ density of rhodopsin in the retina, $\gamma$ is the quantum efficiency of photoisomerization, and $\sigma$ is the surface density of rods (in rods $/ \mathrm{mm}^{2}$ ) at the inner-segment level at which waveguiding into the rods commences. In his investigation of albino and pigmented rat retinas, Low (1987) estimated $D(500 \mathrm{~nm})$, the $\lambda_{\text {max }}$ optical density of rhodopsin in situ, to be 0.313. Data in Lashley (1932), Hagins et al. (1970), and Reiser et al. (in press) provide estimates of the average rod density of the albino rat retina, ranging from $2.5 \times 10^{5}$ to $3.1 \times 10^{5}$ rods/mim ${ }^{2}$; accordingly, $\sigma$ was taken to be $2.7 \times 10^{5}$ rods/mom? Tho quantum efficiency $\gamma$ was taken to have the standard value 0.67 . With these parametcr values, we arrived at the estimate $a_{\mathrm{C}}\left(\lambda_{\max }\right)=1.27 \mu \mathrm{m}^{2}$.

$\Lambda$ more familiar way of estimating $a_{C}$ uses the formula (Baylor et al., 1984; Breton et al., 1994):

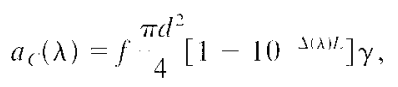

where $d$ is the diameter of the outer segment, $\Delta(\lambda)$ is the specific axial density of rhodopsin in the rod, $L$ is the length of the outer segment, and $f \geq 1$, a dimensionless factor that accounts for any light funneling in the ellipsoid region of the rod inner segment. Typical values in the literature for these parameters appropriate for mammalian rods are $d=1.8-2 \mu \mathrm{m}$, $\Delta\left(\lambda_{\operatorname{mix}}\right)-0.0150 .018 \mu \mathrm{m}$ outer diameter, and $L-2025 \mu \mathrm{m}$, which yields, for $f=1, a_{\mathrm{C}}\left(\lambda_{\max }=0.85-1.35 \mu \mathrm{m}^{2}\right.$. The value calculated in the previous paragraph, therefore, is in reasonable agrecment with the upper linnit calculated with Equation 3 assuming $f=1$. Evidence supports the conclusion that for human rods $f>1$ (Alpern and Pugh, 1974), but nothing is known of relevance in mouse. Breton et al. (1994) estimated $a_{C}\left(\lambda_{\text {mix }}\right)=2.3 \mu \mathrm{m}^{2}$ for human rods, but somewhat arbitrarily set $f=1.8$, implying that the effective diameter of the rod inner segment at the wave-guide aperture was $V f d=2.7 \mu \mathrm{m}$. We doubt that rodent rods could have such a large effective aperture, because even with perfect hexagonal packing of the cllipsoid region of the inner segments, an effective diameter of $2.7 \mu \mathrm{m}$ predicts a maximal rod density of only $1.6 \times$ $10^{5} \mathrm{rods} / \mathrm{mm}^{2}$, well short of the values of $\sigma, 2.5-3.1 \times 10^{5} \mathrm{rods} / \mathrm{mm}^{2}$ cited above. On the other hand, a rod density of $2.5 \times 10^{5} \mathrm{rods} / \mathrm{mm}^{2}$ is consistent with $f=1.3$ and, thus, it is reasonable to think $a_{(}\left(\lambda_{\text {max }}\right)$ could be as high as $1.8 \mu \mathrm{m}^{2}$ in mouse. We will adopt $a_{\mathrm{g}}\left(\lambda_{\max }\right)=1.3 \mu \mathrm{m}^{2}$ for rodent rods as the value most consistent with all of the observations cited in context with Equations 2 and 3.

In summary, we arrived at an estimate of the product of all of the factors multiplying $Q(\lambda)$ in Equation 1 . This product can be taken as an cstimate of the "effective collecting area of a rod at the pupil:" its value is $0.20 \mathrm{\mu m}^{2} / \mathrm{rod}$

\section{Evaluation of scotopic efficacy of broad-band light}

Broad-band ("white") flashes were used to achieve high intensities. These flashes were calibrated for scotopic equivalence with b-wave responses to very $\operatorname{dim} \lambda=500 \mathrm{~nm}$ flashes. Specifically, a serics of highly attenuated white flashes was delivered that cvoked approximately the same amplitude b-wave responses as $500 \mathrm{~nm}$ flashes estimated to produce (1) $=$ $0.1-1.0$. The resulting $b$-wave amplitudes for both white and monochromatic flashes were plotted versus optical density of the filters used for attenuation, and the equivalenec was determined by finding the single factor that shifted the curves for the monochromatic and white flashes into coincidence.

\section{Experimental protocols}

Single-flash protocol. When animals were tested with single flashes, testing was conducted in an ascending order of flash intensities over an intensity range up to $6.5 \log$ units. Sufficient time (up to 5 min for the brightest flashes) was allowed between stimulation for complete dark adaplation Completeness of dark adaptation after stimulation was evaluated by sensitivity of the $b$-wave to flashes estimated with Equation 1 to produce $<1$ photoisomerization per rod (such responses produced no detectable a-waves). These responses were required to achieve the same amplitude before additional flashes in a series were delivered.

Double-flash protocol. The first (test) flash of cach two-flash pair was achromatic and produced $\mid=160-43,000$. The second (probe) flash was it monochromatic flash $(\lambda=501 \mathrm{~nm}, \phi=40,000-50,000)$ delivered by the channel containing the Vivitar flash unit. The intensity of this sceond flash was chosen to produce an essentially saturated a-wave amplitude.

\section{Rod phototransduction cascade model of the a-wave}

The model of the activation phase of the rod G-protein transduction cascade has been described previously in detail and will be presented here only bricfly. Previous work has shown that the normalized circulating current $F(t)$ of amphibian and mammalian rods measured in response to 
brief flashes obeys the relation (Lamb and Pugh, 1992; Pugh and Lamb, 1993):

$$
F(t)=\exp \left[-1 / 2 \Phi A\left(t-t_{\mathrm{eff}}\right)^{2}\right]
$$

where $\Phi$ is the number of photoisomerizations per rod produced by the flash at time $t=0, A$ is a parameter having units of $\mathrm{s}^{-2} / \Phi$, which is characteristic of rods of a given species and which is constant for low-tomoderate intensities, and $t_{\mathrm{eff}}$ is a brief "pure delay" that incorporates the effects of several "microsteps" in the activation reactions. The amplification parameter $A$ can be expressed in terms of the gains of the biochemical activation stages:

$$
A=\nu_{\mathrm{RG}} c_{\mathrm{GP}} \beta_{\mathrm{sub}} n .
$$

$\nu_{\mathrm{RG}}$ is the rate at which a single, fully active $\mathrm{R}^{*}$ activates $\mathrm{G}$-proteins, $c_{\mathrm{GP}}$ is the coupling efficiency between activated G-proteins $\left(G^{*}\right)$ and PDE catalytic subunits (Lamb, 1994), $\boldsymbol{\beta}_{\mathrm{suh}}$ is the rate constant of a single, fully active catalytic subunit of the PDE, and $n$ is the Hill coefficient of the cGMP-activated current. As we will show, this expansion of $A$ into its component gain factors plays an important role in the diagnosis of the mechanism underlying a rapid increase in recovery times for $\Phi \sim 3000$.

The hypothesis linking the measured a-wave component of the ERG, $a(t)$, to the photocurrent response is that the retinal field potential underlying the a-wave is directly proportional to the photocurrent (Hagins et al., 1970; Penn and Hagins, 1972). This linking hypothesis can be expressed as follows:

$$
\begin{aligned}
1-\left[\frac{a(t)}{a_{\max }}\right] & =F(t) \\
& =\exp \left[-1 / 2 \Phi A\left(t-t_{\mathrm{eff}}^{\prime}\right)^{2}\right],
\end{aligned}
$$

where $a(t)$ is the experimentally measured a-wave (in $\mu \mathrm{V}$ ) and $a_{\max }$ is its saturated amplitude. The parameter $t_{\text {eff }}^{\prime}$ is $t_{\text {eff }}$ (compare Eq. 1) augmented by the membrane time constant and hy any delays introduced by the low-pass characteristics of the recording instruments. In the first line of Equation 2, we have made the substitution $t^{\prime}=t-\left(t_{\mathrm{eff}}^{\prime}-t_{\mathrm{eff}}\right)$. Previous work in mammals has shown $t_{\text {eff }}^{\prime}$ to be on the order of a few milliseconds when adequate recording bandwidth is used.

Equation 6 was fitted to the a-wave data with least-squares methods as described in Brelon el al. (1994). Accordingly, $a_{\max }$ first was estimated from the amplitude-saturated responses to intense flashes. Then with $a_{\max }$ fixed the a-wave traces were fitted with Equation 6 with two methods. The first method is "ensemble fitting": for low-to-moderate intensities, entire families of responses were fitted simultaneously with $A$ and varied $t_{\text {eff }}^{\prime}$ to find the best-fitting curve by the least-squares criterion to the entire response family. For our data (see Fig. 3), this method worked extremely well for data sets in which a number of responses for each intensity could be averaged, and also for $\Phi \leqslant 10,000$. Above this latter intensity, both $A$ and $t_{\mathrm{eff}}$ begin to decline, and the traces must be fitted by the "individual fitting" method.

In the individual fitting method, each trace is fitted with Equation 6, allowing both $A$ and $t_{\text {eff }}$ to vary for each trace fitted. This method allows the predicted constancy of $A$ at low-to-moderate intensities to be examined and is nore suitable when the individual traces are single responses (and, therefore, more likely to exhibit some deviation from ideality).

A decline in $\boldsymbol{A}$ and $t_{\mathrm{eff}}$ at higher intensities was expected based on analysis of the biochemical cascade (Lamb and Pugh, 1992) and on earlier investigations of human a-waves (Breton et al., 1994). Nonetheless, even for high intensities Equation 5 has been shown to provide a useful parametric summary of the a-wave traces. In particular, the dependence of $A$ on $\Phi$ can be characterized empirically by a Weber-like saturation function (Breton et al., 1994):

$$
A(\Phi)=\frac{A_{0}}{1+\left(\Phi / \Phi_{1 / 2}\right)},
$$

where $A_{0}$ is the constant value of $A$ at lower intensities, and $\Phi_{1 / 2}$ is the intensity at which $A$ declines to half-maximum. The Gaussian formula, Equation 6, in combination with Equation 7, also yiclds a parametric characterization of the rate-saturation profile.
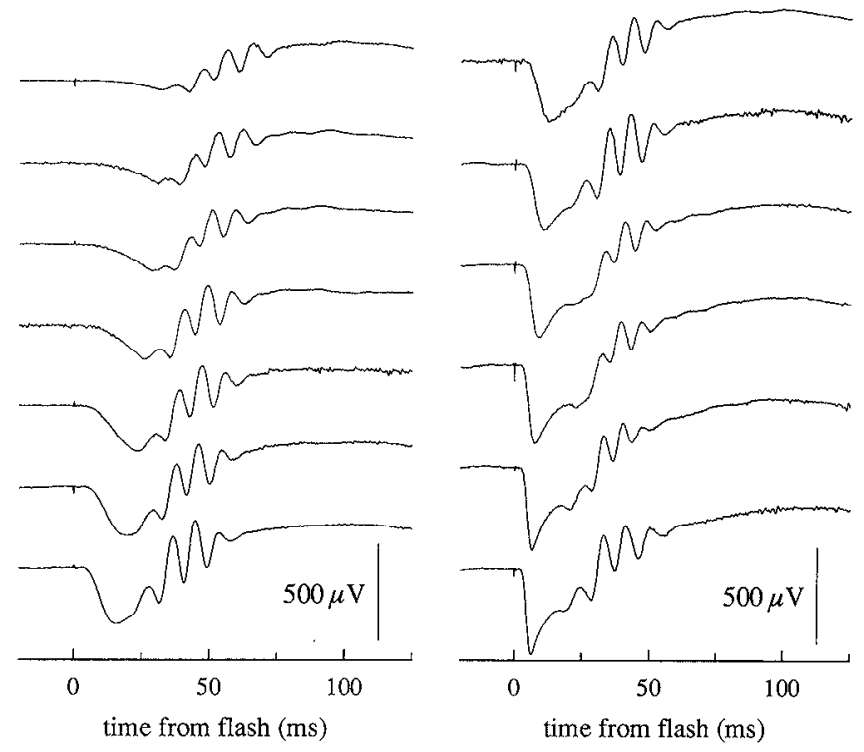

Figure 2. A family of murine ERGs obtained in response to a series of flashes. The flashes were calculated to produce, from dimmest to most intense, $\Phi=54,108,216,432,864,1730,3460,6920,1.38 \times 10^{4}, 2.7 \times 10^{4}$, $5.4 \times 10^{4}, 1.08 \times 10^{5}$, and $2.16 \times 10^{5}$ photoisomerizations per rod. The traces are averages of three to five individual responses. The maximal amplitude, $a_{\max }$, for the response to the most intense flash was $450 \mu \mathrm{V}$.

\section{RESULTS}

\section{Characterization of the activation phase of the murine ERG a-wave}

Figure 2 shows a family of ERGs from a single mouse in a recording session that lasted $\sim 1.5 \mathrm{hr}$. Each record is the average of several flashes. Prominent features include the cornealnegative initial phase (the a-wave, alias fast-PIII), the positivegoing b-wave that follows, and the robust oscillatory potentials. Our concern is with the a-wave, the maximal amplitude of which for the most intense flash for this animal was $450 \mu \mathrm{V}$. For the data of the 11 mice reported here, $a_{\max }=464 \pm 135 \mu \mathrm{V}$ (Table 1 ).

In Figure 3 we replot the a-wave component of each response shown in Figure 2, with the traces now normalized by the amplitude of the response to the most intense flash $\left(\Phi=2.15 \times 10^{5}\right)$. The a-wave traces exhibit both amplitude and rate saturation. The amplitudes of the responses to the two most intense flashes differed by $<5 \%$, and their maximal rates of rise were essentially indistinguishable despite a twofold difference in flash intensity. The data in Figure 3 have been fitted with the theoretical model of the activation phase of the cascade. In this case, the eight responses to flashes producing $\Phi=54-1.38 \times 10^{4}$ were fitted simultaneously with Equation 3: the values of the two parameters characterizing the curves best-fitting these traces were $A=5.6 \mathrm{~s}^{-2}$ and $t_{\text {eff }}^{\prime}=3.6 \mathrm{~ms}$. The apparent values of $A$ and $t_{\mathrm{eff}}^{\prime}$ for the responses to more intense flashes decline; thus, the responses to the more intense flashes were fitted individually rather than as an ensemble. For these cases, the fitted curves provide a convenient way to estimate the maximal rate of rise, $\left(1 / a_{\max }\right) \mathrm{d} a /\left.\mathrm{d} t\right|_{\max }$, as a function of $\Phi$ and to estimate the approach of the maximal rate to its saturated value (see Materials and Methods).

Figure 4 summarizes a body of data obtained from normal mice in which recordings such as those shown in Figure 2 were done, and the analysis of Figure 3 was applied. Figure $4 A$ shows that the maximal rate of rise saturates: the saturated rate of rise is $\sim 600$ $\mathrm{s}^{-1}$, and the intensity that produces the half-saturated rate is 
Table 1. Kinetic parameters of murine a-wave responses

\begin{tabular}{|c|c|c|c|c|c|c|c|c|}
\hline Mouse \# & Protocol & Figures & $\begin{array}{l}a_{\max } \\
(\mu V)\end{array}$ & $\Phi$ (range) & $A_{0}\left(\mathrm{~s}^{-2}\right)$ & $t_{\mathrm{cff}}^{\prime}(\mathrm{ms})$ & $\begin{array}{l}\tau_{\mathrm{c}} \\
(\mathrm{ms})\end{array}$ & $\Phi_{\text {break }}$ \\
\hline $1-5$ & Single-flash & 4 & $408 \pm 83$ & $50-10,000$ & $7.1 \pm 0.7$ & $3.7 \pm 0.6$ & & \\
\hline 6 & Single-flash & $2,3,4$ & 450 & $54-6920$ & 5.6 & 3.6 & & \\
\hline 7 & Double-flash & $4,7,8$ & 350 & $200-6400$ & 6.1 & 3.6 & 260 & 3980 \\
\hline 8 & Double-flash & $4,7,8$ & 470 & $200-3200$ & 9.2 & 4.0 & 210 & 2820 \\
\hline 9 & Double-flash & $4,5,6,7,8$ & 670 & $160-10,200$ & 9.5 & 3.1 & 180 & 3980 \\
\hline 10 & Double-flash & $4,7,8$ & 740 & $160-10,200$ & 8.0 & 2.9 & 250 & 3980 \\
\hline 11 & Double-flash & $4,7,8$ & 380 & $160-10,200$ & 4.7 & 1.9 & 130 & 6310 \\
\hline Mean $\pm \mathrm{SD}$ & & & $464 \pm 135$ & & $7.2 \pm 1.5$ & $3.4 \pm 0.7$ & $206 \pm 53$ & $4210 \pm 1270$ \\
\hline
\end{tabular}

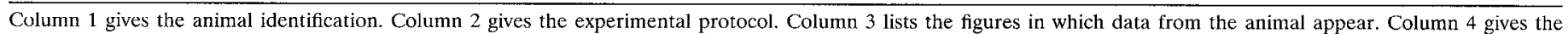

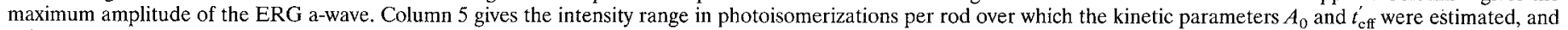

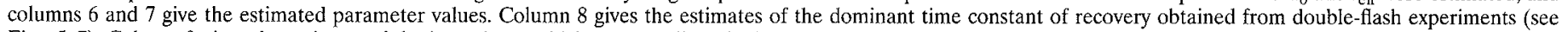

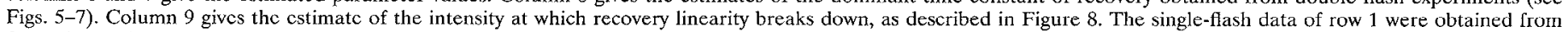

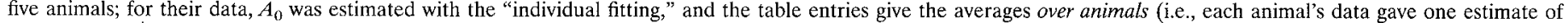
$a_{\mathrm{max}}, A_{0}$, and $\left.t_{\mathrm{eff}}\right)$. All the other estimates were obtained with the "ensemble fitting" method in which the traces were fitted simultaneously.

$\sim 10^{5}$. These values are comparable with those reported previously by Penn and Hagins (1972) for albino rat. Figure $4 B$ shows the estimates of $A$. These estimates were constant for each animal for $\Phi<10,000$, but declined thereafter. Table 1 provides a summary of the results from different animals.

\section{Measurement and characterization of the recovery phase of the a-wave}

Figure 5 illustrates the protocol we used to determine the recovery phase of the photoresponse with pairs of flashes. The top of the figure presents a series of ERGs in which an initial flash produced $\Phi=320$ photoisomerizations; at various times from 0.2 to $1.5 \mathrm{~s}$ after the initial flash, a second, more intense flash $(\Phi=$ $45,000)$ was delivered to probe the residual a-wave amplitude. The

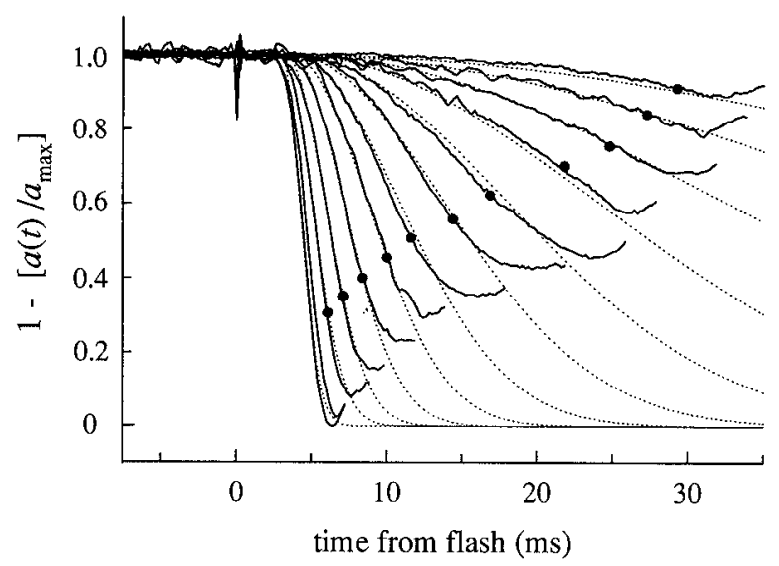

Figure 3. ERG a-wave components from the data of Figure 2 fitted with the transduction cascade activation model. The a-wave components (unbroken, noisy traces) have been extracted from the ERGs of Figure 2 and fitted with the activation model (broken traces). The traces were fitted to minimize the squared deviations from the model (Eq. 6) up to the time points marked with filled circles, except for the responses to the most intense flashes, which were fitted over the entire range from 0 to 1.0. The eight responses to flashes producing $\Psi=54-6920$ photoisomerizations per rod were fitted simultaneously (ensemble fitting method); the parameters of best-fit were $A_{0}=5.6 \mathrm{~s}^{-2}$ and $t_{\text {eft }}^{\prime}=3.6 \mathrm{~ms}$. The remaining four responses were fitted individually with parameters of best-fit $A\left(t_{\mathrm{efff}}^{\prime}\right): 5.55$ $\mathrm{s}^{-2}(3.4 \mathrm{~ms}), 5.1 \mathrm{~s}^{-2}(3.3 \mathrm{~ms}), 4.4 \mathrm{~s}^{-2}(3.1 \mathrm{~ms}), 3.6 \mathrm{~s}^{-2}(3.0 \mathrm{~ms})$, and $2.8 \mathrm{~s}^{-2}$ $(2.9 \mathrm{~ms})$. (For clarity, the theoretical trace fitted to the most intense flash is not shown; it differed only by a very slight translation from that fitted to the next-most intense flash.) The maximal rate of rise of the normalized response to the most intense flash was $476 \mathrm{~s}^{-1}$. intensity of the probe flash was selected so that it produced a near-maximal a-wave amplitude when given alone (probe alone; compare Fig. 2), although at the same time gave a tractable time for complete readaptation. In the bottom of Figure 5, we plot the complement of the residual a-waves (filled circles), which is expected to be proportional to the amplitude of the rod-circulating current. Along with these symbols, we plot two continuous traces of rod responses from mammalian rods obtained in response to flashes estimated by the investigators, who obtained them to

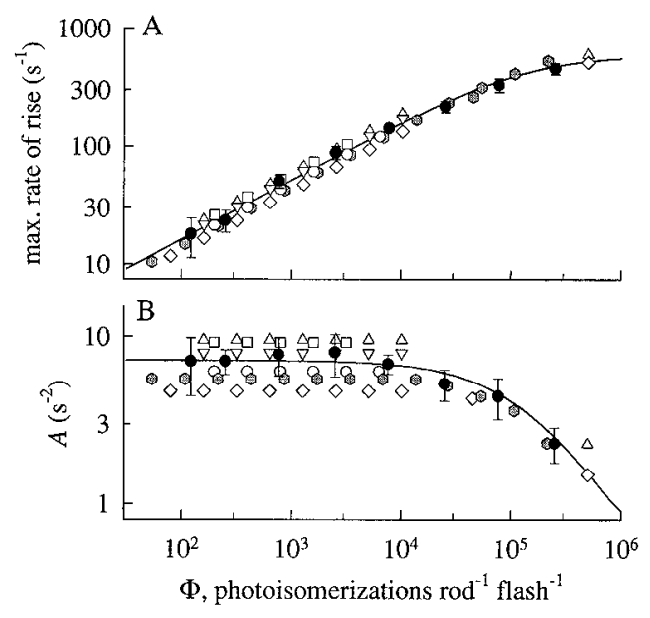

Figure 4. Maximal rates of rise and estimates of amplification coefficients. $A$, Maximal rates of rise of the a-wave components of murine ERGs. Different symbols represent data from different animals. The curve fitted through the data is a rate-saturation function obeying the formula $\mathrm{d} R /\left.\mathrm{d} t\right|_{\max }=\mathrm{d} R /\left.\mathrm{d} t\right|_{\mathrm{sat}}\left[\Phi /\left(\Phi+\Phi_{1 / 2}\right)\right]^{1 / 2}$, where $R(t)=a(t) / a_{\max }$, with $\mathrm{d} R /\left.\mathrm{d} t\right|_{\text {sat }}=600 \mathrm{~s}^{-1}$ and $\Phi_{1 / 2}=1.36 \times 10^{5}$; this relation can be derived from Equations 6 and 7. $B$, Estimates of the amplification coefficients of a-wave data. Different symbols represent data of different mice. Points plotted below $\Phi=10,000$ that lie on a straight line were estimated with the ensemble fitting method, in which an entire family of a-waves was fit simultaneously as in Figure 3. The larger open symbols with the dots in the center represent the averaged data of five different animals, the a-wave traces of which were fitted individually; the error bars are $\pm 1 \mathrm{SD}$. The theoretical curve drawn through the data is a Weber-saturation function given by Equation 7 , characterized by the parameters $A_{0}=7.2 \mathrm{~s}^{-2}$ (constant value at lower intensities) and $\Phi_{1 / 2}=1.36 \times 10^{5}$. The points in $A$ and $B$ are in 1:1 correspondence according to the Gaussian formula (Eq. 6). However, $A$ emphasizes the rate-saturation behavior, whereas $B$ emphasizes the constancy of the amplification coefficients at low-to-moderate intensities. 


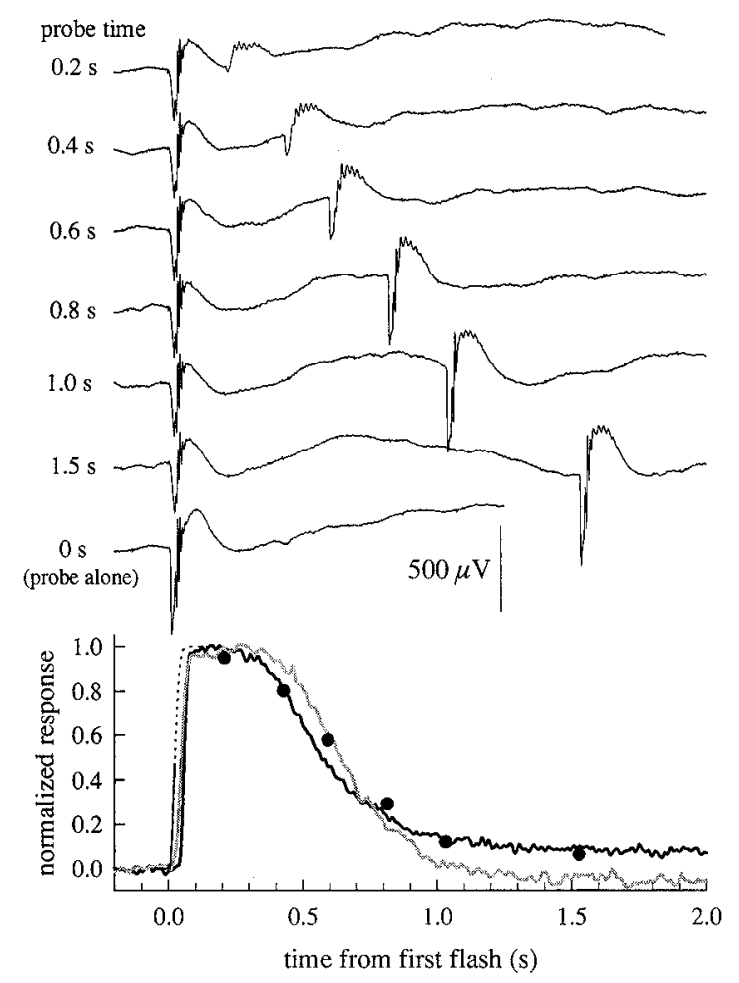

Figure 5. Top, ERG records from a portion of a double-flash experiment on one animal. An initial flash producing $\Phi_{1}=320$ photoisomerizations per rod was followed by a second probe flash $\left(\Phi_{2}=45,000\right)$ at $0.2,0.4,0.6$, $0.8,1.0$, or $1.5 \mathrm{~s}$. The lowest trace shows the response to the probe flash alone; complete recovery of $\mathbf{b}$-wave sensitivity to very dim flashes delivered after the probe flash took $<1 \mathrm{~min}$. Bottom, Reconstruction of the recovery phase. The filled circles show the recovery phase of the a-wave reconstructed from the traces at the top; each point plots $1-\left[a\left(t_{\text {probe }}\right)\right]$ $\left.a_{\text {max }}\right]$, i.e., the complement of the amplitude of the response to the probe flash at the time point $t=t_{\text {probe }}$, normalized by the amplitude of the probe flash alone-that is, the probe delivered to a fully dark-adapted animal. Note that a single time axis is used for both the top and bottom. Shown as the thinner black solid line is the averaged a-wave trace from the data in the top, normalized as usual; the dotted line shows the predicted extension of the a-wave trace obtained by the fitting of Equation 6 to the activation phase, as in Figure 3. The thicker gray line is taken from the recordings of Penn and Hagins (1972) of isolated rat retinas at $36^{\circ} \mathrm{C}$ and was estimated by them to produce $\Phi=300$ (the trace was obtained by scanning the published figure). The thicker black trace is taken from the recordings of Baylor et al. (1984; their Fig. 1) of an isolated monkey rod; it is estimated to have produced $\Phi=417$ based on an assumed side-on collecting area of $2 \mu \mathrm{m}^{2}$. (The entire family of traces from which this latter trace was taken was provided by Dr. Schnapf, University of California, San Francisco.)

produce approximately the same value of $\Phi$ as the initial flash delivered to the mouse.

In Figure 6 we present a family of response recoveries obtained with the two-flash protocol illustrated in Figure 5 in the same experimental session. The intensities of the initial flashes ranged from $\Phi=160$ (leftmost data set, open circles) to $\Phi=20,200$ (rightmost data set, filled triangles); we replot the data from the bottom of Figure 5 (black-filled circles). For flashes estimated to produce $\Phi=2560$ or less, the response recoveries are isomorphic, i.e., they have the same shape but are translated on the time axis. The isomorphic recoveries also obey a quantitative law in which geometric increments in $\Phi$ produce linear increments in the recovery half-times.

This latter quantitative law is illustrated in Figure 7 , in which the recovery half-times are plotted as a function of $\Phi$ on a semilog plot for five animals. Here we have fitted straight lines to the

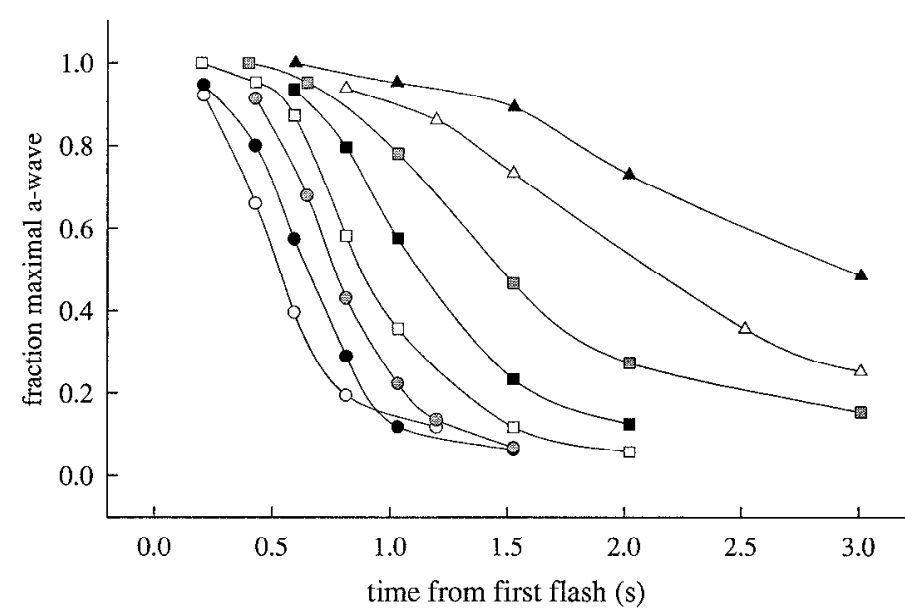

Figure 6. An entire family of reconstructed response recoveries. Data points (symbols) show eight members of a family of a-wave response recoveries to initial flashes reconstructed in the same manner as illustrated in Figure 5 and producing, from left to right: $\Phi=160 ; 320 ; 640 ; 1280$; $2540 ; 5080 ; 10,100$; and 20,200 . The data points have been connected by cubic splines. (These data are from the experiment of Fig. 5; the data points from the bottom of that figure are repeated as the second set from the left in this figure.) Note that the response recoveries to the four least-intense flashes are isomorphic but translated on the time axis and that subsequent recoveries have a prolonged time course.

recovery half-time data of animals over the intensity region corresponding to the isomorphic recoveries.

\section{DISCUSSION}

\section{Reconstruction of the murine rod photoresponse from the ERG}

Our results show that it is possible to recover both the activation and inactivation phases of the murine rod photoresponse from the a-wave component of the ERG. Whereas the identification of the a-wave with the activation phase of the rod response has becn accepted for many years (Penn and Hagins, 1972), only recently has its activation kinetics been characterized in terms of models of the rod response (Hood and Birch, 1990a,b, 1994; Breton et al., 1994), and only very recently has the recovery phase been reconstructed from the human ERG (Birch et al., 1995). Our analysis has extended the investigation of Birch et al. (1995) in the following ways: (1) by exanining the recovery time courses to moderate intensity flashes at interflash intervals of $<1 \mathrm{~s}$ (Figs. 5, 6); (2) by providing evidence of consistency of the shape of the recovery with published mammalian photocurrent responses at comparable values of $\Phi$ (Fig. 5); and (3) by demonstrating that the recovery kinetics is isomorphic for moderate values of $\Phi$ that do not saturate the initial a-wave amplitude but that are expected to saturate the amplitude of the rod photocurrent (Fig. 6).

Application of the cascade model allowed us to characterize the activation phase of the murine a-wave quantitatively. We found the amplification constant, $A_{0}$, to have the value $7.2 \pm 1.5 \mathrm{~s}^{-2} / \Phi$. A previous analysis of the photoreceptor literature estimated $A_{0}$ $=4.0-9.8 \mathrm{~s}^{-2}$ from published photocurrent records of isolated mammalian rods (Pugh and Lamb, 1993). In fact, analysis of the entire family of monkey rod responses from which the thick black trace in Figure 5 was extracted yielded the value $A_{0}=7.6 \mathrm{~s}^{-2}$ (Pugh and Lamb, 1993) (Fig. 8). Estimates of $A_{0}$ based on normal human a-wave data have ranged from 4 to $7 \mathrm{~s}^{-2}$ (Cideciyan and Jacobson, 1993; Breton et al., 1994; Hood and Birch, 1994). 


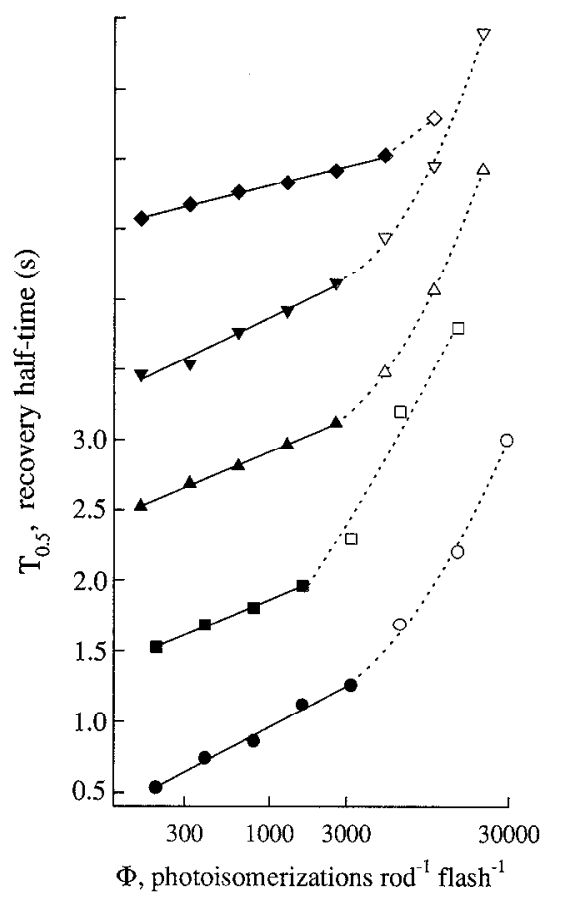

Figure 7. Estimation of a dominant time constant of recovery for moderate-intensity flashes from a-waves of five mice. Semilog plot of $T_{0.5}$, the recovery half-time, versus $\Phi$. Each plotted point represents the value of $T_{0.5}$ estimated from a complete recovery curve as in Figure 5. Each connected data set (one set of symbols) represents the set of midpoints of recovery for an entire family of recoveries (as in Fig. 6) from one animal. The filled symbols were fitted with a linear regressions (solid lines) to estimate $\tau_{c}$, the dominant time constant of recovery (the estimates of $\tau_{c}$ are given in Table 1). The points of data that deviate from the linear regression line (open symbols) have been connected with a higher-order regression curve (broken lines). The bottom set of data points is in its correct position with respect to the ordinate; for clarity, the other curves have been offset upward in increments of $2.0 \mathrm{~s}$ except for the top set, which was offset an additional $0.25 \mathrm{~s}$. The same symbols are used for the data of each animal in this plot and in Figures 5 and 8; the animals are listed as numbers $7-11$ in Table 1.

\section{The dominant time constant of murine rod photoresponse recovery}

A body of data is consistent with the view that over certain intensity regimes photoresponse recovery kinetics is rate-limited, or "dominated," by an inactivation mechanism having a first-order decay (Baylor et al., 1974; Adelson, 1982; Hodgkin et al., 1984; Pepperberg et al., 1992). Two necessary conditions for the extraction of a rate-limiting time constant from recovery curves are as follows: (1) recoveries must obey translation invariance over some intensity regime; and (2) the incremental translation per geometric increase in $\Phi$ must be a constant (for review, see Adelson, 1982). Our data show that these two conditions are met (Figs. 6 , 7 ). Thus, over the range $\Phi \sim 160-2500$, our results are consistent with a dominant recovery mechanism having a time constant $\tau_{c}$ $\sim 200 \mathrm{~ms}$. Our estimate of $\tau_{\mathrm{c}}$ in mouse is consistent with previous estimates in human (Adelson, 1982; Birch et al., 1995) over approximately the same intensity range.

\section{Effective lifetime of $\mathbf{R}^{\star}$}

Based on data obtained in recordings of human ERGs, Birch et al. (1995) have argued that the lifetime of $R *$ is $\sim 2 \mathrm{~s}$. Because the dominant time constant provides an estimate of the intermediate with the longest lifetime in a reaction cascade, it is clear that for $\Phi$
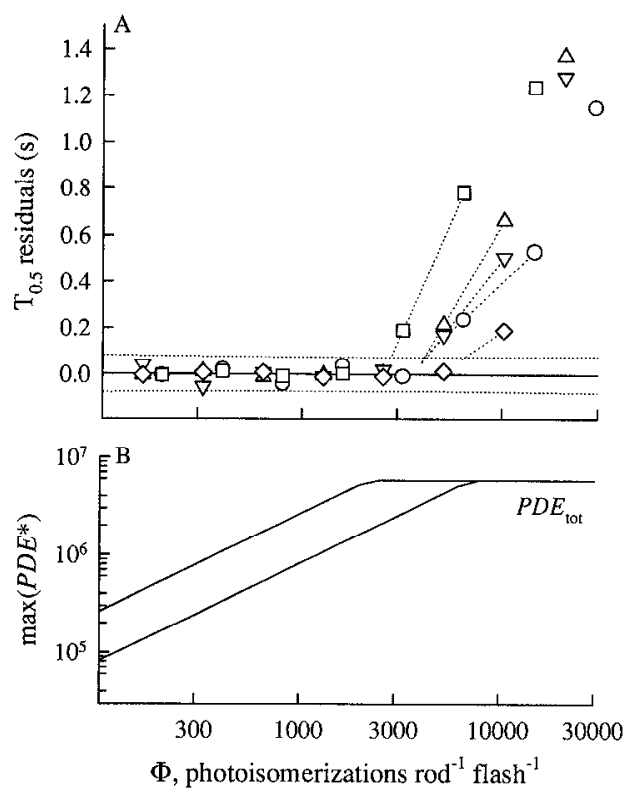

Figure 8. Estimation of the intensity of breakdown of recovery linearity and prediction of the GAP hypothesis. $A$. The residuals of all of the recovery half-time data from the linear regressions of Figure 7 are shown. The residuals have been refitted with a linear regression over the intensity region up to $\Phi=2500$. This later regression curve (solid line) had a slope insignificantly different from zero; a $95 \%$ confidence band around the line also is shown. The first two points of each set of residuals that are above the confidence band have becn fittcd with a linear regression, and the intensity $\Phi_{\text {break }}$ at which "recovery linearity" breaks down has been estimated as the point at which these two-point regressions intercept the upper confidence-band limit (for the data set represented by diamonds, only one point is above the confidence band and, in this case, the line was fitted to it and its nearest neighbor from the data set; triangles, squares, and circles represent data from different animals). $B$, Double-logarithmic plot showing calculations of the maximum number of $\mathrm{PDE}^{*}$ activated by flashes of the intensity indicated on the abscissa. The calculations were done with Equation 8 . The value of $\nu_{\mathrm{RP}}$, the rate at which each $\mathrm{R}^{*}$ activates PDEs, was estimated from the expression $\nu_{\mathrm{RP}}=A_{0} / \beta_{\text {sub }} n$, where $A_{0}=7.2 \mathrm{~s}^{-2}$ is the grand mean of all estimates of $A_{0}$ in Table 1 (see Fig. $4 B), \beta_{\text {sub }}=4 \times 10^{-4}$ is the rate constant of a single PDE catalytic subunit in a volume equal to the mouse rod outer segment aqueous space, and $n$ $=2$ is the Hill cocfficient of the cGMP-activated conductance (sce Eq. 5). Predicted lines (slope $=1$ ) are shown for two different values of $\tau_{\mathrm{R}^{*}}$, the effective lifetime of the enzymatically active form of rhodopsin: the line to the left is for $\tau_{\mathrm{R}}{ }^{*}=290 \mathrm{~ms}$; the line to the right is for $\tau_{\mathrm{R}}{ }^{*}=90 \mathrm{~ms}$. The intersection of these curves with the ordinate value $\mathrm{PDE}^{*} \equiv \mathrm{PDE}_{\text {tot }}$, the total number of PDE catalytic subunits in the outer segment, is the value of $\Phi_{\text {break }}$ predicted by the hypothesis that each $G^{*}$ must bind to an unoccupied $\mathrm{PDE}_{\gamma}$ for its terminal phosphate to be hydrolyzed.

$<3000$ the average lifetime of $R^{*}$ cannot exceed 200 ms by much in mice.

\section{One account for prolonged recoveries: long-lived but rare $\mathbf{R}^{*}$}

We offer two hypotheses to account for the prolonged recoveries that occur for $\Phi>3000$. The first hypothesis is based on the work of Baylor et al. (1984), who reported that monkey rod photocurrents exhibit prolonged, step-like recovery events for flashes producing $\Phi>250-2000$, depending on the rod. They hypothesized that such events are caused by the low probability of production of $\mathrm{R} *$ with much longer than normal lifetimes. Thus, if in mouse rods $\sim 1$ in $3000 R^{*}$ has a long lifetime, then flashes producing $\Phi>$ 3000 should exhibit recoveries prolonged relative to what is expected if $\tau_{c}$ always were $200 \mathrm{~ms}$. 


\section{Alternative account for prolonged recoveries: the GTPase-activating hypothesis}

Recent investigations have shown that the $\mathrm{PDE}_{\gamma}$ is a GTPaseactivating (GAP) factor (Arshavsky and Bownds, 1992; Arshavsky et al., 1994), i.e., hydrolysis of the terminal phosphate of $\mathrm{G}^{*}$ $\left(\mathrm{G}^{*} \equiv \mathrm{G} \alpha-\mathrm{GTP}\right)$ occurs much more rapidly when $\mathrm{G}^{*}$ is bound to $\mathrm{PDE}_{\gamma}$. Conversely, because the hydrolysis of its terminal phosphate is slower when a $G^{*}$ is not bound to $\operatorname{PDE}_{\gamma}$, when $G^{*}$ are produced in excess of the total number of $\mathrm{PDE}_{\gamma}$ subunits, response recovery is expected to be prolonged until uncomplexed $\mathrm{G}^{*}$ can find a free $\mathrm{PDE}_{\gamma}$ and bind to it. We now inquire whether this GAP hypothesis can predict the intensity at which the isomorphism of the mouse response recoveries breaks down and recoveries become much slower.

In Figure 8 we illustrate calculations comparing our data with the prediction of the GAP hypothesis. In Fig. $8 A$ we plot the residuals from the regression lines of the recovery half-time data of Figure 7 . For $\Phi \leq 2560$, these residuals were refitted with a straight line. A $95 \%$ confidence band has been drawn around this regression line, and both line and bands have been extrapolated across the intensity axis. We estimated the intensity $\Phi_{\text {break }}$ at which the residuals for each data set rise above this confidence hand as that value of $\Phi$ at which a straight line through the first two points above the band intercepts the upper confidence band. The mean \pm SD of the five estimates of $\Phi_{\text {break }}$ determined in this manner is $4210 \pm 1270$ (Table 1 , last column); the $90 \%$ confidence interval for $\Phi_{\text {break }}$ is $(2220,7480)$.

In Fig. $8 B$ we plot two lines illustrating the maximum number of activated PDE-catalytic subunits per rod [PDE*; Fig. $8 B$, $\left.\max \left(P D E^{*}\right)\right]$ for flashes of different intensities, calculated with the following formula:

$$
\max \left(\mathrm{PDE}^{*}\right)_{\Phi} \sim \Phi \nu_{\mathrm{RP}} \tau_{\mathrm{R}^{*}}
$$

This formula is a direct generalization of the "ramping" behavior predicted for PDE activation (Lamb and Pugh, 1992; Lamb, 1991). In Equation $8, \tau_{R}{ }^{*}$ is the effective lifetime $R^{*}$ and $\nu_{R P}$ is the effective rate of production of $\mathrm{PDE}^{*}$ by a single, fully active $\mathrm{R}^{*}$. We estimated the value of $\nu_{\mathrm{RP}}$ from our data as $\nu_{\mathrm{RP}}=A_{0} / \beta_{\mathrm{sub}} n$ (Eq. 5). The rate constant $\beta_{\text {sub }}$ has been estimated from biochemical and biophysical data of mammalian rods to be $4 \times 10^{-4} \mathrm{~s}^{-1}$ (Pugh and Lamb, 1993; their Table 2), and the Hill coefficient of the cGMP-activated channels is taken to be $n=2$ (Fesenko et al., 1985; Yau and Baylor, 1989; Koutalos and Yau, in press) Thus, based on our average value $A_{0}=7.2 \mathrm{~s}^{-2}$ (Table 1), we estimate $\nu_{\mathrm{RF}}=9000 \mathrm{PDF}^{*}$ per fully activated $\mathrm{R}^{*}$ per second. The value $\nu_{\text {RP }}=9000$ is approximately two- to threefold higher than expected from light-scattering analysis of G-protein activation in bovine rod membranes (Hofmann and Kahlert, 1991). Hofmann and Kahlert (1991) estimated $\nu_{\mathrm{RG}}$, the maximal rate of $\mathrm{G}^{*}$ production by a single $\mathrm{R}^{*}$, to be $4000 \mathrm{~s}^{-1}$ at $37^{\circ} \mathrm{C}$; thus, if $c_{\mathrm{GP}}$, the coupling efficiency between $\mathrm{G}^{*}$ and $\mathrm{PDE}^{*}$, is $\sim 0.7$ (Lamb, 1994), the results of Hofmann and Kahlert (1991) would predict $\nu_{\mathrm{KP}}=$ $\nu_{\mathrm{RG}} c_{\mathrm{GP}} \sim 3000$. Our estimate of $\nu_{\mathrm{RP}}$ could be reconciled with that of Hofmann and Kahlert (1991) if the product $\beta_{\text {sub }} n$ were threefold higher.

The simplest form of the GAP hypothesis predicts $\Phi_{\text {break }}$ to be that intensity at which the total complement of PDE-catalytic subunits, $P D E_{\text {tot }}$, is activated. Assuming there is one holo-PDE for each 50 rhodopsin molecules (Sitaramayya ct al., 1986) and two catalytic subunits and two $\gamma$ subunits for each holo-PDE, a $25-\mu \mathrm{m}-$ long mammalian rod having $1.4 \times 10^{8}$ rhodopsin molecules should have
$P D E_{\mathrm{tot}}=5.8 \times 10^{6}$. Thus, the GAP hypothesis predicts $\Phi_{\text {break }}$ to be the value at which the unity-sloped lines of Fig. $8 B$ intersect the ordinate value $5.8 \times 10^{6}$. Rearranging Equation 8 , we have:

$$
\tau_{\mathrm{R}^{*}}=\frac{P D E_{\mathrm{tot}}}{\Phi_{\mathrm{break}} \nu_{\mathrm{RP}}} .
$$

Taking $\Phi_{\text {break }}=4210$ and $\nu_{\mathrm{RP}}=9000$, we arrive at the estimate $\tau_{\mathrm{R}}{ }^{*}=150 \mathrm{~ms} . \Lambda \mathrm{n}$ approximate confidence interval for $\tau_{\mathrm{R}}{ }^{*}$ can be obtained by inserting the $90 \%$ upper and lower confidence limits for $\Phi_{\text {break }}$ into Equation 9; this yields the interval $(90,290)$ in milliseconds, which includes the dominant time constant $\tau_{\mathrm{c}}=210$ $\mathrm{ms}$. The confidence interval is approximate because it depends on estimates of several factors, including and especially $\beta_{\text {sub }}, n$, and $P D E_{\text {tot. }}$. Certainly, the hypothesis that all $\mathrm{R}^{*}$ have a lifetime as long as $1-2$ s for $\Phi<3000$ can be excluded.

\section{Long-lived $R^{\star}$ and excessive $G^{\star}$ combined as explanation of prolonged recoveries}

The two explanations of the prolonged recoveries offered above are not mutually exclusive. Each mammalian rod disk face contains 1600 holo-PDE, and even if $\nu_{\mathrm{RP}}$ were as low as $3000 \mathrm{~s}^{-1}$, an $\mathrm{R}^{*}$ with a lifetime of $>1$ s should produce complete activation of the 3200 PDE catalytic subunits on its disk face, and an excess of $\mathrm{G}^{*}$. Such complete local activation of the PDE is a likely component of a comprehensive explanation of the step-like photocurrent events observed by Baylor et al. (1984) in their recordings of monkey rods, and by Chen et al. (1995) in their recordings of rods of mice expressing a mutant rhodopsin lacking the C-terminal region that contains phosphorylation sites thought to be essential to normal $\mathrm{R}^{*}$ inactivation.

\section{REFERENCES}

Adelson EH (1982) 'The delayed rod afterimage. Vision Res 22:1313-1328.

Alpern M, Pugh Jr EN (1974) The density and photosensitivity of human rhodopsin in the living retina. J Physiol (Lond) 237:341-370.

Alpern M, Fulton AB, Baker BN (1987) "Self-screening" of rhodopsin in rod outer segments. Vision Res 27:1459-1470.

Arshavsky VY, Bownds MD (1992) Regulation of deactivation of photoreceptor G-protein by its target enzyme and cGMP. Nature $357: 416-417$.

Arshavsky VY, Dumke CL, Zhu Y, Artemyev NO, Skiba NP, Hamm HE, Bownds MD (1994) Regulation of transducin GTPase activity in bovine rod outer segments. J Biol Chem 269:19882-19887.

Baylor DA, Hodgkin AL, Lamb TD (1974) The electrical response of turtle cones to flashes and steps of light. J Physiol (Lond) 242:685-727.

Baylor DA, Lamb IU, Yau K-W (19/9) 'I he membrane current of single rod outer segments. J Physiol (Lond) 288:589-611.

Baylor DA, Nunn BJ, Schnapf JL (1984) The photocurrent, noise and spectral sensitivity of rods of the monkey, Macaca fascicularis. J Physiol (Lond) 357:575-607.

Birch DG, Fish GE (1987) Rod ERGs in retinitis pigmentosa and conerod degeneration. Invest Ophthalmol Vis Sci 28:140-150.

Birch DG, Hood DC, Nusinowitz S, Pepperberg DR (1995) Abnormal activation and inactivation mechanisms of rod transduction in patients with autosomal dominant retinitis pigmentosa and the Pro-23-His $\mathrm{Mu}$ tation. Invest Ophthalmol Vis Sci 36:1603-1614.

Breton ME, Schueller AW, Lamb TD, Pugh Ji EN (1994) Analysis of ERG a-wave amplification and kinetics in terms of the G-protein cascade of phototransduction. Invest Ophthalmol Vis Sci 35:295-309.

Brown KT, Watanabe K, Murakami M (1965) The early and late receptor potentials of monkey cones and rods. Cold Spring Harbor Symp Quant Biol 30:457-482.

Carter-Dawson RD, LaVail MM (1979) Rods and cones in the mouse retina. I. Structural analysis using light and electron microscopy. J Comp Neurol 188:245-262. 
Chen J, Makino CL, Peachey NS, Baylor DA, Simon MI (1995) Mechanisms of rhodopsin inactivation in vivo as revealed by a $\mathrm{COOH}$-terminal truncation mutant. Science 267:374-377.

Cideciyan A, Jacobson S (1993) Negative electroretinograms in retinitis pigmentosa. Invest Ophthalmol Vis Sci 34:3253-3263.

Fesenko EE, Kolesnikov SS, Lyubarsky AL (1985) Induction by cyclic GMP of cationic conductance in plasma membrane of retinal rod outer segments. Nature 313:310-313.

Frishman LJ, Steinberg RH (1989) Intraretinal analysis of the threshold dark-adapted ERG of cat retina. J Neurophysiol 61:1221-1232.

Goto Y, Peachey NS, Ripps H, Naash MI (1995) Functional abnormalities in trans-genic mice expressing a mutant rhodopsin gene. Invest Ophthalmol Vis Sci 36:62-71.

Green DG (1973) Scotopic and photopic components of the rat electroretinogram. J Physiol (Lond) 228:781-797.

Hagins WA, Penn RD, Yoshikami S (1970) Dark current and photocurrent in retinal rods. Biophys J 10:380-412.

Hodgkin AL, McNaughton PA, Nunn BJ, Yau K-W (1984) Effect of ions on retinal rods from Bufo marinus. J Physiol (Lond) 350:649-680.

Hofmann KP (1995) Light-induced protein-protein interactions on the rod photoreceptor disc membrane. In: Biomembranes (Lee ed). New York: JAI, in press.

Hood DC, Birch DG (1990a) The a-wave of the human electroretinogram and rod receptor function. Invest Ophthalmol Vis Sci 31: $2070-2081$.

Hood DC, Birch DG (1990b) A quantitative measure of the electrical activity of human rod photoreceptors using electroretinography. Vis Neurosci 5:379-387.

Hood DC, Birch DG (1992) A computational model of the amplitude and implicit time of the b-wave of the human ERG. Vis Neurosci $8: 107-126$.

Hood DC, Birch DG (1994) Rod phototransduction in retinitis pigmentosa: estimation and interpretation of parameters derived from the rod a-wave. Invest Ophthalmol Vis Sci 35:2948-2961.

Kraft TW, Schneeweis DM, Schnapf JL (1993) Visual transduction in human rod photoreceptors. J Physiol (Lond) 464:747-765.
Koutalos Y, Yau K-W (1995) $\mathrm{Ca}^{2+}$ modulation of the cyclic GMP-gated channel of bullfrog retinal rod photoreceptors. J Physiol (Lond), in press.

Lamb TD (1994) Stochastic simulation of activation in the G-protein cascade of phototransduction. Biophys J 67:1439-1454.

Lamb TD, Pugh Jr EN (1992) A quantitative account of the activation steps involved in phototransduction in amphibian photoreceptors. J Physiol (Lond) 449:719-757.

Lashley KS (1932) The mechanism of vision. J Comp Psychol 13:173-200.

Low JC (1987) Rhodopsin levels in the isolated normal and $+r d$ mouse retina. Ophthalmic Res 19:49-51.

Penn RD, Hagins WA (1972) Kinetics of the photocurrent of retinal rods. Biophys J 12:1073-1094.

Pepperberg DR, Cornwall MC, Kahlert M, Hofmann KP, Jin J, Jones GJ, Ripps H (1992) Light-dependent delay in the falling phase of the retinal rod photoresponse. Vis Neurosci 8:9-18.

Pugh Jr EN, Lamb TD (1993) Amplification and kinetics of the activation steps in phototransduction. Biochim Biophys Acta 1141:111-149.

Reiser MA, Williams TP, Pugh Jr EN (1996) The effect of light history on the aspartate-isolated fast-PIII responses of the albino rat retina. Invest Ophthalmol Vis Sci 37:221-229.

Remtulla S, Hallett PE (1985) A schematic eye for the mouse, and comparisons with the rat. Vision Res 25:21-31.

Schnapf JL, Nunn BJ, Meister M, Baylor DA (1990) Visual transduction in cones of the monkey, Macaca fascicularis. J Physiol (Lond) 427:681-713.

Sitaramayya A, Harkness J, Gonzalez-Olivia C, Liebman PA (1986) Kinetic studies suggest that light-activated cyclic GMP phosphodiesterase is a complex with G-protein subunits. Biochemistry 25:651-656.

Yau K-W (1994) Phototransduction mechanism in retinal rods and cones. Invest Ophthalmol Vis Sci 35:9 32.

Yau K-W, Baylor DA (1989) Cyclic GMP-activated conductance of retinal photoreceptor cells. Annu Rev Neurosci 12:289-327. 